\title{
The Impact of Gabapentin Administration on Brain GABA and Glutamate Concentrations: A 7T 'H-MRS Study
}

\author{
Kejia Cai ${ }^{1,5}$, Ravi PR Nanga ${ }^{1,5}$, Lisa Lamprou ${ }^{2,3}$, Claudia Schinstine ${ }^{2,3}$, Mark Elliott', Hari Hariharan', \\ Ravinder Reddy' and C Neill Epperson*,2,3,4 \\ 'Department of Radiology, Center for Magnetic Resonance and Optical Imaging (CMROI), Perelman School of Medicine at the University \\ of Pennsylvania, Philadelphia, PA, USA; ${ }^{2}$ Department of Psychiatry, Perelman School of Medicine at the University of Pennsylvania, \\ Philadelphia, PA, USA; ' Department of Psychiatry, Penn Center for Women's Behavioral Wellness, Perelman School of Medicine at the \\ University of Pennsylvania, Philadelphia, PA, USA; ${ }^{4}$ Department of Obstetrics and Gynecology, Perelman School of Medicine at the \\ University of Pennsylvania, Philadelphia, PA, USA
}

Gamma-aminobutyric acid (GABA) and glutamate are implicated in numerous neuropsychiatric and substance abuse conditions, but their spectral overlap with other resonances makes them a challenge to quantify in humans. Gabapentin, marketed for the treatment of seizures and neuropathic pain, has been shown to increase in vivo GABA concentration in the brain of both rodents and humans. Gabapentin effects on glutamate are not known. We conducted a gabapentin ( $900 \mathrm{mg}$ ) challenge in healthy human subjects to confirm and explore its effects on GABA and glutamate concentrations, respectively, and to test the ability of single voxel localized proton magnetic resonance spectroscopy ( ${ }^{H} \mathrm{H}-\mathrm{MRS}$ ) to reliably measure GABA and glutamate in the visual cortex at the ultra-high magnetic field of 7 Tesla. Reproducibility of GABA and glutamate measurements was determined in a comparison group without drug twice within day and 2 weeks apart. Although GABA concentration changes were small both within day (average 5.6\%) and between day (average 4.8\%), gabapentin administration was associated with an average increase in GABA concentration of 55.7\% (6.9-91.0\%). Importantly, druginduced change in GABA levels was inversely correlated to the individual's baseline GABA level $\left(R^{2}=0.72\right)$. Mean glutamate concentrations did not change significantly with or without drug administration. In conclusion, localized 'H-MRS at 7 Tesla can be successfully applied to the measurement of GABA concentration and is sensitive to acute drug-induced changes in cortical GABA. Whether baseline GABA concentrations predict clinical efficacy of gabapentin is an area worthy of exploration. Neuropsychopharmacology (2012) 37, 2764-277I; doi:I0.1038/npp.2012.142; published online 8 August 2012

Keywords: gabapentin; 7 Tesla; $\gamma$-aminobutyric acid; GABA; glutamate; Glu

\section{INTRODUCTION}

Gamma-aminobutyric acid (GABA), the major neurotransmitter for fast inhibitory synaptic transmission and tonic inhibitory control, is present in $25-50 \%$ of all synapses (Sutoo et al, 2000). L-glutamic acid (glutamate) is responsible for fast excitatory neurotransmission and is present at approximately $80 \%$ of brain synapses (Sutoo et al, 2000). GABA is synthesized from glutamate by removal of a $\alpha$ carboxyl group by glutamic acid decarboxylase (GAD). The synthetic relationship between GABA and glutamate suggests that concentrations of each may be influenced by the other. Their widespread presence and utilization among

*Correspondence: Dr C Neill Epperson, Penn Center for Women's Behavioral Wellness, 3535 Market Street, Room 300I, Philadelphia, PA 19104, USA, Tel: + I 215 573-887I, Fax: + I 215573 888I,

E-mail: cepp@mail.med.upenn.edu

${ }^{5}$ These authors contributed equally to this work.

Received 13 April 2012; revised 27 June 2012; accepted 28 June 2012 neurons in the brain implicates both GABA and glutamate in practically all functions of the central nervous system, as well as in a wide-range of neuropsychiatric and substance abuse conditions, making them two of the most promising targets for pharmacologic drug development.

In humans, GABA and glutamate naturally occur in the range of 0.8 to $1.8 \mathrm{mM}$ and 8 to $10 \mathrm{mM}$, respectively, and can be difficult to detect in vivo because of complicated spectral patterns and overlap with other resonances. Proton magnetic resonance spectroscopy $\left({ }^{1} \mathrm{H}-\mathrm{MRS}\right)$ is a safe, noninvasive method for repeated measure of brain GABA and glutamate in human subjects that does not require radioactive tracers or dyes. ${ }^{1} \mathrm{H}-\mathrm{MRS}$ has been applied to the study of GABA and/or glutamate in seizure disorders (Petroff et al, 1995, 1996a,b), major depression (Price et al, 2009; Hasler et al, 2007; Sanacora et al, 1999, 2004; Auer et al, 2000), anxiety disorders (Hasler et al, 2009; Goddard et al, 2001), premenstrual dysphoric disorder (Epperson et al, 2002), schizophrenia (Kegeles et al, 2012; Marsman et al, 2011), bipolar disorder 
(Benedetti et al, 2009), nicotine, alcohol, and cocaine addiction (Epperson et al, 2006; Gomez et al, 2011; Licata and Renshaw, 2010) and neurodegenerative diseases such as Parkinson's Disease and Alzheimer's Disease (Griffith et al, 2008). The majority of published in vivo human ${ }^{1} \mathrm{H}-\mathrm{MRS}$ studies have been conducted at fields of 4 Tesla or less and have not included within day or between day reproducibility data for either GABA or glutamate.

${ }^{1} \mathrm{H}$-MRS conducted at the ultra-high magnetic field of 7 Tesla has the advantage of detecting amino-acid neurotransmitters with greater sensitivity than is possible at 3 or 4 Tesla. GABA resonances overlap with those of glutamate, $\mathrm{N}$-acetylaspartic acid (NAA), creatine, and macromolecules. To study the role of GABA and GABA-modulating drugs in the pathophysiology and treatment of brain disorders it is crucial to maximize the signal-to-noise ratio (SNR) and document reproducibility of GABA-editing methods. To date, only one group has reported GABA measurements using MEGA editing at 7 Tesla (Terpstra et al, 2002) focusing on methods for macromolecule suppression and not reproducibility or response to pharmacologic manipulation.

Gabapentin (1-(aminomethyl)cyclohexaneacetic acid; Neurontin) is approved by the United States Food and Drug Administration for the treatment of partial seizures (Honarmand et al, 2011) and postherpetic neuralgia (Edelsberg et al, 2011). In addition, gabapentin has been found to be effective in prevention of frequent migraine headaches (Mathew et al, 2001) and other pain syndromes (Dallocchio et al, 2000; Rapchuk et al, 2010). Gabapentin has been studied in bipolar disorder with less than optimal results (Cipriani et al, 2011). Gabapentin is a lipophilic compound that was initially synthesized to mimic the chemical structure of GABA by addition of a cyclohexyl to its backbone (Honarmand et al, 2011). Although it is rapidly absorbed, readily crosses the blood-brain barrier and is orally active in several animal models of epilepsy, gabapentin neither binds to $\mathrm{GABA}_{\mathrm{A}}$ or $\mathrm{GABA}_{\mathrm{B}}$ receptors nor is it metabolized to GABA (Goa and Sorkin, 1993; Kammerer et al, 2011; Taylor et al, 1992). Research regarding gabapentin's effects on GABA and glutamate synthetic and metabolizing enzymes reveals a complex pattern of activity and provides an incomplete explanation for its anticonvulsant effects. Gabapentin stimulates GAD at drug concentrations of 1.0 to $2.5 \mathrm{mM}$ (Silverman et al, 1991; Taylor et al, 1992) and inhibits the GABA-catabolizing enzyme, GABA-transaminase (GABA-T) at high concentrations (23-25 mM; Taylor et al, 1992) with relatively weak effects on GABA-T at lower concentrations of $10 \mathrm{mM}$ (Goldlust et al, 1995). Gabapentin increased GABA turnover in 11 out of 12 brain regions tested, but the temporal effect of the drug varied from region to region (Loscher et al, 1991). Other possible mechanisms of action include inhibition of neuronal calcium influx (Fink et al, 2000), binding to $\alpha_{2} \delta$ calcium channel subunits (Gee et al, 1996) and activation of glutamate dehydrogenase (Cho et al, 1998).

Although the mechanism of gabapentin therapeutic action is unclear, human subjects studies suggest that its administration leads to an overall increase in central GABA levels. In neocortical tissue extracted from individuals with intractable epilepsy, gabapentin administration resulted in $13 \%$ increase in cellular GABA concentrations (Errante et al, 2002). Humans studied at $4.1 \mathrm{~T}$ and $2.1 \mathrm{~T}$ experienced an average $48 \%$ and $55 \%$ increase in occipital cortex GABA concentrations, respectively, when gabapentin was administered (Kuzniecky et al, 2002; Petroff et al, 1996b). Several preclinical studies implicate neuronal (Mathews and Diamond, 2003) and astrocyte (Heja et al, 2009) glutamate uptake in the regulation of GABA synthesis and release. However, the impact of GABA-enhancing drugs on glutamate concentrations in vivo has not been previously investigated. Hence, the goal of the investigation described herein was to develop reliable ${ }^{1} \mathrm{H}$-MRS methods for GABA and glutamate quantification at $7 \mathrm{~T}$ that provide highly reproducible data and are sensitive to the detection of acute pharmacologic-induced changes in neurotransmitter levels.

\section{MATERIALS AND METHODS}

\section{Subjects}

Eleven healthy non-smoking males (mean \pm SD age; $27.5 \pm 7.2$ years) underwent ${ }^{1} \mathrm{H}$-MRS pre- and $2.5 \mathrm{~h}$ post-oral gabapentin administration (900 mg). The majority of the sample was Caucasian, two subjects were African American and one was Hispanic. Subjects were excluded if they met criteria for a present or past diagnosis of a psychiatric or substance abuse disorder according to the Structured Clinical Interview for Diagnosis for the DSM-IV, Non-Patient Version (First et al, 2002). Subjects were also excluded if they had a positive urine toxicology, metallic implant, history of claustrophobia, a significant medical or neurological illness or use of any psychotropic medications within the previous 12 months. All subjects gave written informed consent for participation in this study protocol, which was approved by the institutional review board at the Perelman School of Medicine of the University of Pennsylvania. A subset of the subjects $(n=6)$ who completed the gabapentin administration also participated in a study of the reproducibility of the MEGA-PRESS GABA-editing sequence (Bielicki et al, 2004; Mescher et al, 1998). Ten subjects in total were scanned twice in the same day without drug administration, 13 were scanned twice 2 weeks apart. Scans were typically $60 \mathrm{~min}$ in duration and subjects relaxed under research staff supervision between scans on gabapentin test days.

\section{MRS of GABA and Glutamate}

All the experiments were performed on a Siemens 7T whole body scanner with a vendor supplied circularly polarized volume RF coil. Localized manual shimming of the $\mathrm{B}_{0}$ field was performed on a brain voxel in the visual cortex (12cc, Figure 1a) to obtain localized water line width of $\sim 0.1$ p.p.m. or less.

The acquisition protocol consists of a gradient echo localizer, a 3D Magnetization Prepared RApid Gradient Echo (MPRAGE) whole brain acquisition, 3D reformatting of the MPRAGE data and single voxel spectroscopy acquisitions from a voxel placed in the midline of the visual cortex. To minimize or avoid lipid contamination in the spectroscopy, the voxel was placed approximately $1 \mathrm{~cm}$ away from skull. For reproducibility studies, we used the MPRAGE images and custom-built voxel co-registration software derived from SPM to position voxels consistently between scans. 

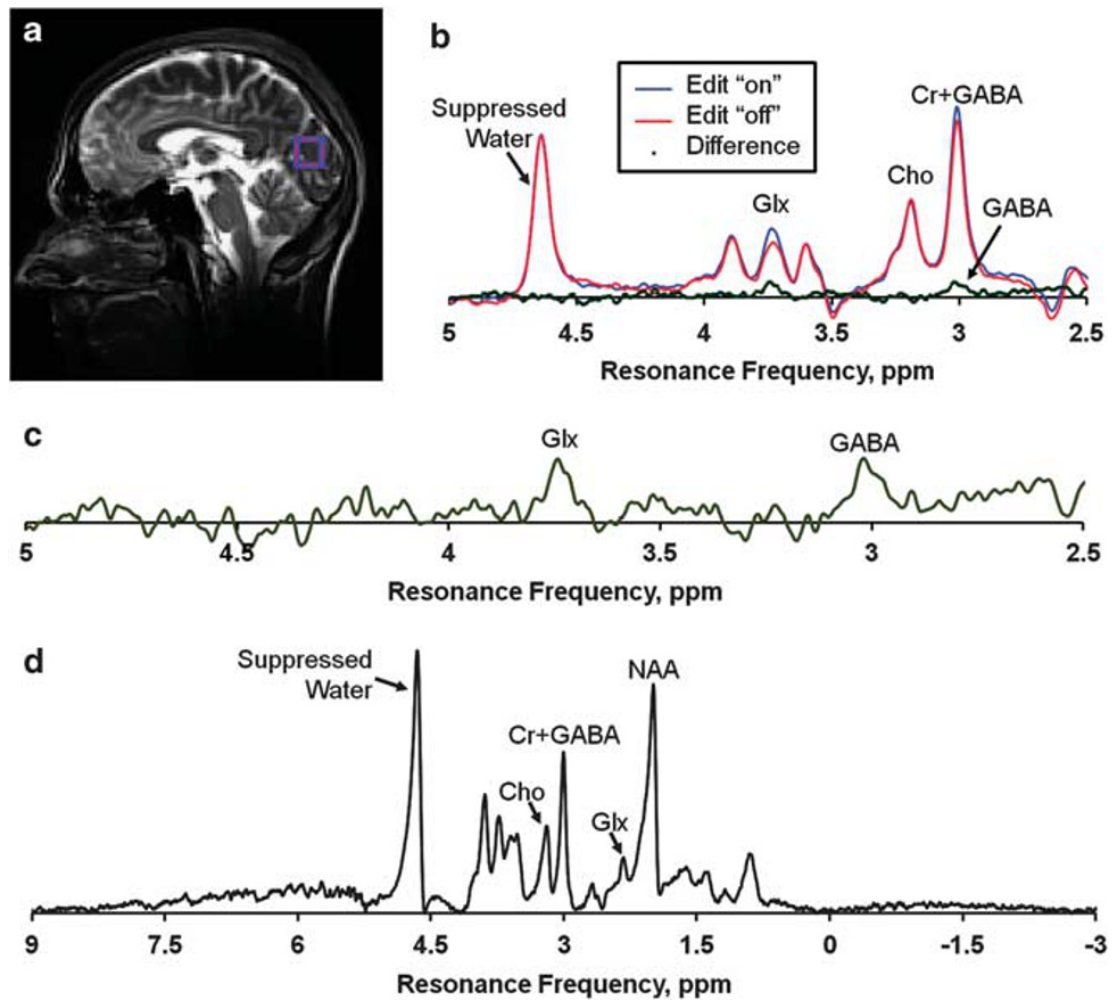

Figure I (a) Localization of the visual cortex voxel is indicated as a box in the sagittal T2-weighted brain image. (b) A representative set of GABA spectra with edit 'on' (blue), 'off (red) and their difference (green dots). (c) Rescaled difference spectrum (green) shows GABA signal at 3 p.p.m. and Glx peak located at $\sim 3.75$ p.p.m. (d) A representative water suppressed short-echo spectrum used for quantification of glutamate. Cho, choline; Cr, creatine; Glx, glutamate + glutamine; NAA, N-acetylaspartate.

GABA-edited single voxel spectra (SVS) were obtained with a custom-modified Point-RESolved Spectroscopy (PRESS) sequence with MEGA-PRESS GABA-editing pulses (Bielicki et al, 2004; Mescher et al, 1998) that are frequency selective either at 1.9 p.p.m. to the $\mathrm{GABA}-\mathrm{CH}_{2}$ protons attached to C2 (edit 'on') or at 7.5 p.p.m. (edit 'off') and using following parameters: spectral width $=4 \mathrm{kHz}$, number of points $=2048$, averages $=100$, echo time $(\mathrm{TE})=0.069 \mathrm{~s}$ and repetition time $(\mathrm{TR})=3 \mathrm{~s}$. Water suppression was achieved with a variable power RF pulses with optimized relaxation delays (VAPOR) (Tkac et al, 1999), which was played out before the SVS acquisition. Global and local baselines were removed using a recently published algorithm (Cobas et al, 2006) with manual setting of parameters. Macromolecular peaks centered at 3.8, 3.6, 1.9, 1.7, 1.5, 1.2, 1.0 , and 0.7 p.p.m. were fitted for removing macromolecule contamination. Total acquisition time was $10 \mathrm{~min}$.

Short TE SVS were obtained with a PRESS sequence using following parameters: spectral width $=4 \mathrm{kHz}$, number of points $=2048$, averages $=100, \mathrm{TE}=20 \mathrm{~ms}$ and $\mathrm{TR}=3 \mathrm{~s}$. Water suppression was achieved with VAPOR, which was played out before the SVS acquisition. Total acquisition time was $5 \mathrm{~min}$. Water reference spectra was acquired by using short TE $(20 \mathrm{~ms})$ SVS with water suppression turned 'off' and 16 averages for total acquisition time of $48 \mathrm{~s}$.

\section{MRS Quantification}

Custom-built spectroscopy processing packages were developed in MATLAB (R2007b, MathWorks, Natick, MA). In general, SVS spectra were processed from the raw free induction decay data by exponential apodization of $10 \mathrm{~Hz}$ for glutamate and $20 \mathrm{~Hz}$ for GABA to remove GABA spinspin splitting, Fourier transformation, phase correction, and baseline removal, followed by least-squared curve fitting as sum of Gaussian peaks.

GABA signals are buried under the creatine peak at 3 p.p.m. Subtraction of the two spectra with editing pulse 'off and 'on' provides GABA signals (Figure $1 \mathrm{~b}$ ) from $-\mathrm{CH}_{2}$ protons attached to C3. GABA signal was then fitted as a single Gaussian function, whose integral was normalized to the creatine integral from the edit 'off spectrum for quantification of GABA concentration assuming $8 \mathrm{mM}$ creatine concentration in the brain (Choi et al, 2011; Rothman et al, 1984, 1993; Detre et al, 1991).

For glutamate quantification, water suppressed SVS were fitted as a sum of Gaussian peaks manually positioned at locations corresponding to brain metabolites. The integration of 2.35 p.p.m. glutamate peak was normalized by water reference signal for glutamate quantification.

\section{Statistical Analysis}

One-way analysis of variance was performed using MATLAB to compare the differences in GABA and glutamate concentrations within day, between day, pre- and post-drug administration. Values are reported as mean $\pm \mathrm{SD}$. Group differences were considered to be significant at the $p<0.05$ level. Pearson's correlation analysis was performed to examine the relationship between change in GABA 
concentration with gabapentin administration and baseline GABA levels.

\section{RESULTS}

\section{MEGA-PRESS GABA Editing}

As depicted in Figure 1b, MEGA-PRESS GABA-edited sequences using a frequency selective inversion pulse on GABA $-\mathrm{CH}_{2}$ at 1.9 p.p.m. decouples the J-coupling between its triplet at 3 p.p.m., creating higher signal at 3 p.p.m. The GABA-edited blue spectrum has higher amplitude than the edit 'off' spectra shown in red. Subtraction of these two spectra gives the GABA peak at $\sim 3$ p.p.m.

\section{Effects of Acute Gabapentin Administration}

All 11 subjects completed the pre- and post-gabapentin scans without adverse effects although post-gabapentin data were lost from one subject because of motion during spectrum acquisition during the post-drug administration scan. Most subjects reported either no discernable effect of the drug or simply a feeling of being calm or mildly sleepy. Figure 2 shows representative GABA spectra where the GABA peak was estimated as a Gaussian function pre (Figure 2a) and post (Figure $2 \mathrm{~b}$ ) oral administration of gabapentin. GABA elevation post-drug administration is readily observed. Mean \pm SD pre- and post-gabapentin concentrations were $0.96 \pm 0.28$ and $1.43 \pm 0.25 \mathrm{mM}$, respectively (Figures $3 \mathrm{a}$ and 4a). As seen in Figure 3b, linear fitting of this variation as a function of the GABA concentration of the pre-gabapentin scan shows that the magnitude of change in GABA
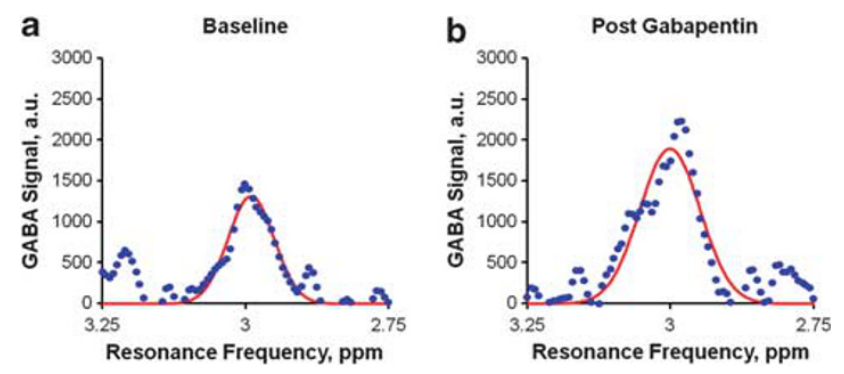

Figure 2 A representative set of pre- (a) and 2.5-h (b) post-gabapentin GABA spectra. Elevation of GABA peak is clearly observable.
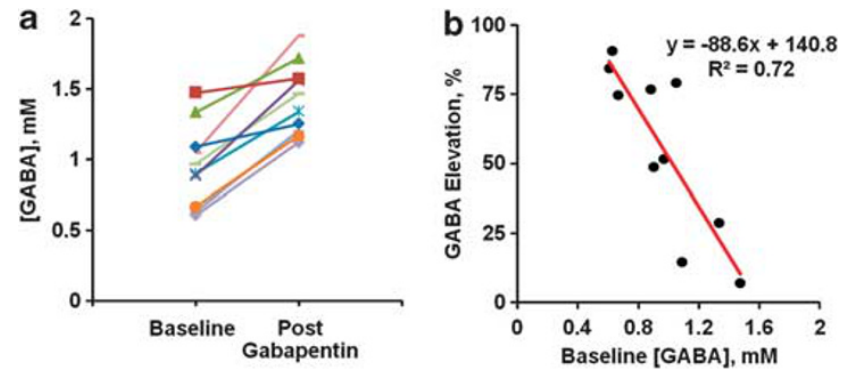

Figure 3 Individual's GABA concentrations of the two scans in the gabapentin group (a). Lines with different colors represent different subjects. GABA percentage change was modeled as a linear function of the baseline GABA concentration (b). concentration was inversely correlated with baseline GABA levels $\left(y=-88.6 \mathrm{x}+140.8, R^{2}=0.72\right)$. Mean $\pm \mathrm{SD}$ percent change in GABA concentrations from the pre- to postgabapentin scan was $55.7 \pm 30.3 \%$ with concentration increases ranging from a low of $6.9 \%$ to a high of $91.0 \%$ (Figure 4a). This is in stark contrast to the small percent change (5.6 \pm 3.2$)$ observed within day when no gabapentin was administered $(\mathrm{F}=26.9 ; p<0.0001$, Figure $4 \mathrm{~b})$.

Reproducibility of our GABA-editing methods is demonstrated by the similarity in mean GABA concentration between scans conducted in the same individual within day $(0.99 \pm 0.36$ and $1.05 \pm 0.34 \mathrm{mM}$, Figure $4 \mathrm{~b})$ and between day $(1.10 \pm 0.18$ and $1.13 \pm 0.33 \mathrm{mM}$, data not shown). For those six men who also underwent gabapentin administration, the percent change in GABA within day without drug was similar $(5.3 \pm 3.5 \%)$ to the entire group $(5.6 \pm 3.2 \%)$. There was little correlation between change in GABA concentration within day and levels from the first within day scan when gabapentin was not administered $(y=-6.5 x+10.0$, $R^{2}=0.16$ ). Data were lost because of motion on one within day scan.

Glutamate data were not obtained on 2 of the 11 subjects undergoing gabapentin challenge in order to shorten the scan session $(n=2)$. In two of the other subjects, the glutamate data suffered from motion artifact during that portion of the scan. Subjects underwent glutamate measurement within $(n=9)$ and between day $(n=8)$ without gabapentin administration to examine reproducibility of our methods. Glutamate concentrations (Figure 5) were $11.19 \pm 0.91 \mathrm{mM}$ before gabapentin administration and $11.10 \pm 1.69 \mathrm{mM}$ postadministration, representing a mean percent difference of $-2.92 \pm 14.64 \%$ (ranges from -25.6 to $+18.1 \%$ ). Within
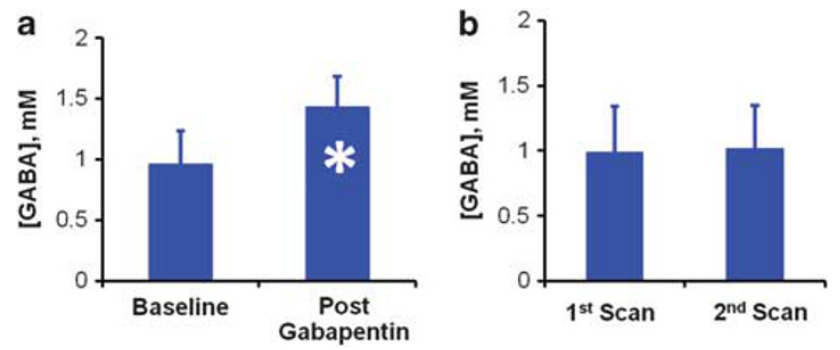

Figure 4 Average GABA concentrations of the two scans in the gabapentin (a) and control (b) studies. Significant GABA elevation is seen $2.5 \mathrm{~h}$ after oral gabapentin dose $(* p<0.05)$.
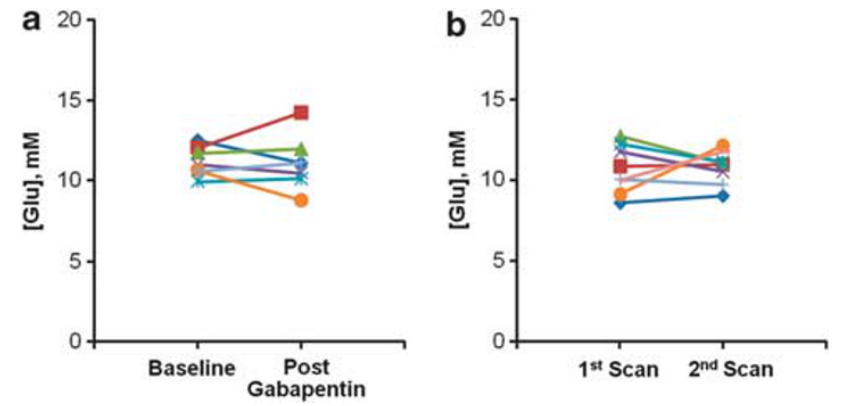

Figure 5 Individual's glutamate concentrations of the two scans in the gabapentin (a) and control (b) groups. Lines with different colors represent different subjects. 
day glutamate levels were $10.65 \pm 1.49 \mathrm{mM}$ for scan 1 and $10.79 \pm 1.03 \mathrm{mM}$ and for scan 2 with an average variation $11.48 \pm 8.87 \%$ (ranges from 1.26 to $28.19 \%$ ). Between day glutamate levels were $11.39 \pm 1.11 \mathrm{mM}$ on day 1 and 10.96 $\pm 1.43 \mathrm{mM}$ on day 2 with an average variation $6.56 \pm 4.69 \%$ (ranges from 1.43 to $13.49 \%$ ).

\section{DISCUSSION}

Our finding that gabapentin acutely and robustly increases visual cortex GABA concentrations in most healthy subjects is consistent with two smaller studies, one conducted in healthy controls scanned at 4.1T (Kuzniecky et al, 2002) and one conducted in individuals with epilepsy scanned at 2.1T (Petroff et al, 1996b). Similar to findings from Petroff et al, (1996a), we detected, on average, a $0.84 \mathrm{mM}$ increase in GABA concentration with acute gabapentin administration, the magnitude of increase being inversely correlated with baseline GABA concentrations (Petroff et al, 2000). Although this study was conducted in healthy controls, these data are relevant as previous studies have found little correlation between antiepileptic (gabapentin, topirimate, and vigabatrin) dose and seizure control (Petroff et al, 2006). In contrast, the larger the increase in GABA concentration with acute administration of vigabatrin, a GABA transaminase inhibitor, the greater the seizure control in epileptic subjects (Petroff et al, 1999). Whether the same relationship exists between seizure control and gabapentin induced increase in GABA concentrations has not been studied.

Not surprisingly, there appears to be a ceiling with respect to how high GABA concentrations can be pushed with antiepileptic administration and at which there is no additional benefit with respect to seizure control (Petroff et al, 1996a, c). In our study, the highest GABA concentration obtained with gabapentin administration was $1.88 \mathrm{mM}$, representing a $79 \%$ increase from baseline. In other studies of healthy subjects and those with epilepsy, GABA concentrations were as high as 2.3 and $2.7 \mathrm{mM}$, respectively, within hours of ingesting gabapentin $1200 \mathrm{mg}$ (Kuzniecky et al, 2002; Petroff et al, 2000). The differences in final postdrug levels between our study and others is likely due to differences in baseline GABA levels, ours being lower, and variations in GABA concentrations reported from studies conducted a different magnetic field strengths.

Another important finding is that MEGA-PRESS GABAediting methods can be successfully applied to the acquisition of GABA spectra with sufficient SNR to allow for reliable quantification of GABA concentration. With an average $<6 \%$ difference in GABA concentrations both within day and between day, we are capable of detecting smaller differences between groups (Epperson et al, 2002, 2006; Sanacora et al, 1999) or with pharmacologic and nonpharmacologic interventions than those previously observed (Sanacora et al, 2002, 2008).

Although these data are compelling, there are several limitations in the generalizability of our findings. The most obvious is that we did not include women in this study. Our group has previously demonstrated menstrual cycle phase differences in GABA concentrations in healthy women (Epperson et al, 2002) and for this reason we chose to focus on males as they would be expected to have less day-to-day variation in GABA concentrations (Epperson et al, 2006). We could have chosen to study all women in the same menstrual cycle phase. However, the early to midfollicular phase would have been the most ideal as ovarian hormones are consistently low, but this is the time when healthy women tend to have their highest GABA concentrations (Epperson et al, 2002), perhaps limiting our detection of drug-induced changes in GABA concentration. Sex difference in GABA response to AEDs has not been previously explored and deserves full investigation with women being studied in both follicular and luteal phases of the menstrual cycle.

Our initial in vivo quantification using the LC model (O'Gorman et al, 2011; Provencher, 1993) for the reproducibility studies were inconsistent. In addition, the LC model phantom basis for 7T is not readily available. Hence, our data processing relies on custom-developed processing packages. With this approach, we have obtained consistent quantification of metabolites from within day scans of control subjects.

The detection of GABA resonance using the editing method can be complicated by overlap from macromolecules. However at $7 \mathrm{~T}$, the editing pulse becomes more selective because of the increased frequency separation between GABA and macromolecules. Based on the previous study on macromolecular contamination at 7T (Terpstra et al, 2002), we estimate that macromolecules contribute about 15 to $30 \%$ to the estimated GABA concentration in our study. However, macromolecule contamination remains a challenge for accurate quantification of brain metabolites using short echo-time spectroscopy. Methods currently under development include using double inversion recovery for metabolite-nulling to obtain macromolecular spectra, which can be used for subtraction or as prior knowledge for fitting (Kassem and Bartha, 2003; Mader et al, 2002). Further validation may be needed to test efficiency of macromolecule removal using these methods.

Although gabapentin administration resulted in an increase in GABA concentrations in all subjects, the glutamate response was considerably more variable and there was no overall mean difference in glutamate concentrations between pre- and post-drug administration. However, we cannot rule out that chronic doses of gabapentin would have had a consistent effect on glutamate concentrations as an acute dose failed to alter glutamate concentrations while twice daily dosing for 8 days led to a decrease in glutamate in a rodent model (Leach et al, 1997). It is possible that healthy individuals have a narrow homeostatic range for GABA concentrations as reflected by the $<6 \%$ difference in within day and between day values, whereas there is a relatively larger range in glutamate concentration across and between days that is physiologic given the subjects included in this study were free from psychiatric and substance abuse disorders. One potential source of variability between scans conducted on the same day is caffeine withdrawal. Subjects are fasting between scans in a given day and acute caffeine administration increases glutamate concentrations in some brain regions (Solinas et al, 2002). Glutamate acquisition occurred at the end of each scan session and the quality of the data could have been affected by subject fatigue with having to 
remain motionless. However, we lost data from only two subjects because of motion artifact.

Finally, what do these data suggest about the relationship between GABA and glutamate in response to treatment with this novel antiepileptic drug? With acute gabapentin dosing, the increase in GABA is predicated on the baseline GABA concentration. Whether chronic gabapentin treatment, which is required for its therapeutic effect, has a similar relationship with baseline GABA concentration is not known. Rodent studies would suggest that gabapentin enhances GAD activity at clinically relevant drug levels, whereas much higher drug levels than are typically obtained in the clinical setting are required to inhibit GABA-T (Silverman et al, 1991; Taylor et al, 1992). Both drug effects would be expected to lead to an overall increase in GABA concentration similar to that observed in this study. However, gabapentin also reduces calcium influx into glutamatergic terminals contributing to a decrease in glutamate release (Fink et al, 2000), However, chronic treatment would be necessary most likely to observe an overall change in GABA concentration because of decreased release of glutamate. Moreover, inhibition of calcium influx leads to reduced cytosolic calcium and overall neuronal excitability (Fink et al, 2000), suggesting gabapentin's antiepileptic mechanism of action could have little to do with acute increases in GABA concentration. Investigation of acute and chronic effects of gabapentin on both GABA and glutamate, particularly in brain regions typically implicated in seizure disorders, would be required to further examine gabapentin's anti-seizure effects in human subjects.

In conclusion, with the largest group to date, we demonstrated GABA-enhancing effects of gabapentin administration in healthy subjects. Glutamate concentrations measured during the same scan did not show a characteristic pattern of response to drug administration and were consistent with findings when no drug was administered. Whether each individual has a tightly controlled homeostatic GABA concentration and relatively greater variability in glutamate concentrations from day-to-day needs to be confirmed in a larger sample. Regardless, these data suggest that future studies focusing on glutamate concentrations in human subjects should account for day-to-day variability and include at least two baseline measurements for each subject. The same does not seem to be true for GABA concentrations, at least not in the visual cortex. Finally, ${ }^{1} \mathrm{H}$-MRS conducted at $7 \mathrm{~T}$ provides an exquisitely sensitive tool for quantification of GABA and glutamate in human subjects at baseline and with pharmacologic manipulation.

\section{ACKNOWLEDGEMENTS}

We thank Jacqueline Meeks and Norman Butler from Hospital of University of Pennsylvania for their help with subject scanning, Drs Singh Anup and Haris Mohammad for their constructive suggestions, Sidyarth Garimall for his assistance in brain image segmentation. This work was supported by the National Center for Research Resources and the National Institute of Biomedical Imaging and Bioengineering of the National Institutes of Health through grant number P41-EB015893 and R21-DA032256.

\section{DISCLOSURE}

Dr Epperson receives funding from the NIH. In addition, she is the recipient of an investigator initiative grant from Shire and product donation for research purposes from Novartis and has stock in Merck and Johnson and Johnson. Dr Reddy receives funding from the NIH. The remaining authors declare no conflict of interest.

\section{REFERENCES}

Auer DP, Pütz B, Kraft E, Lipinski B, Schill J, Holsboer F (2000). Reduced glutamate in the anterior cingulate cortex in depression: an in vivo proton magnetic resonance spectroscopy study. Biol Psychiatry 47: 305-313.

Benedetti F, Calabrese G, Bernasconi A, Cadioli M, Colombo C, Dallaspezia S et al (2009). Spectroscopic correlates of antidepressant response to sleep deprivation and light therapy: a 3.0 Tesla study of bipolar depression. Psychiatry Res 173: 238-242.

Bielicki G, Chassain C, Renou JP, Farges MC, Vasson MP, Eschalier A et al (2004). Brain GABA editing by localized in vivo (1) $\mathrm{H}$ magnetic resonance spectroscopy. NMR Biomed 17: 60-68.

Cho SW, Cho EH, Choi SY (1998). Activation of two types of brain glutamate dehydrogenase isoproteins by gabapentin. FEBS Lett 426: 196-200.

Choi C, Ganji SK, DeBerardinis RJ, Dimitrov IE, Pascual JM, Bachoo $\mathrm{R}$ et al (2011). Measurement of glycine in the human brain in vivo by $1 \mathrm{H}-\mathrm{MRS}$ at $3 \mathrm{~T}$ : application in brain tumors. Magn Reson Med 66: 609-618.

Cipriani A, Barbui C, Salanti G, Rendell J, Brown R, Stockton S et al (2011). Comparative efficacy and acceptability of antimanic drugs in acute mania: a multiple-treatments meta-analysis. Lancet 378: 1306-1315.

Cobas JC, Bernstein MA, Martin-Pastor M, Tahoces PG (2006). A new general-purpose fully automatic baseline-correction procedure for 1D and 2D NMR data. J Magn Reson 183: 145-151.

Dallocchio C, Buffa C, Mazzarello P, Chiroli S (2000). Gabapentin vs. amitriptyline in painful diabetic neuropathy: an open-label pilot study. J Pain Symptom Manage 20: 280-285.

Detre JA, Wang ZY, Bogdan AR, Gusnard DA, Bay CA, Bingham $\mathrm{PM}$ et al (1991). Regional variation in brain lactate in Leigh syndrome by localized $1 \mathrm{H}$ magnetic resonance spectroscopy. Ann Neurol 29: 218-221.

Edelsberg JS, Lord C, Oster G (2011). Systematic review and metaanalysis of efficacy, safety, and tolerability data from randomized controlled trials of drugs used to treat postherpetic neuralgia. Ann Pharmacother 45: 1483-1490.

Epperson CN, Gueorguieva R, Czarkowski KA, Stiklus S, Sellers E, Krystal JH et al (2006). Preliminary evidence of reduced occipital GABA concentrations in puerperal women: a $1 \mathrm{H}-\mathrm{MRS}$ study. Psychopharmacology (Berl) 186: 425-433.

Epperson CN, Haga K, Mason GF, Sellers E, Gueorguieva R, Zhang $\mathrm{W}$ et al (2002). Cortical gamma-aminobutyric acid levels across the menstrual cycle in healthy women and those with premenstrual dysphoric disorder: a proton magnetic resonance spectroscopy study. Arch Gen Psychiatry 59: 851-858.

Errante LD, Williamson A, Spencer DD, Petroff OA (2002). Gabapentin and vigabatrin increase GABA in the human neocortical slice. Epilepsy Res 49: 203-210.

Fink K, Meder W, Dooley DJ, Gothert M (2000). Inhibition of neuronal $\mathrm{Ca} 2+$ influx by gabapentin and subsequent reduction of neurotransmitter release from rat neocortical slices. $\mathrm{Br} \mathrm{J}$ Pharmacol 130: 900-906.

First MB, Spitzer RL, Gibbon M, Williams JBW. Structured Clinical Interview for DSM-IV-TR Axis I Disorders, Research Version, Non-Patient Edition. (SCID-I/NP). Biometrics Research, New York State Psychiatric Institute: New York. November 2002. 
Gee NS, Brown JP, Dissanayake VUK, Offord J, Thurlow R, Woodruff GN (1996). The novel anticonvulsant drug, gabapentin (Neurontin), binds to the $\alpha_{2} \delta$ subunit of a calcium channel. J Biol Chem 271: 5768-5776.

Goa KL, Sorkin EM (1993). Gabapentin: A review of its pharmacological properties and clinical potential in epilepsy. Drugs 46: 409-427.

Goddard AW, Mason GF, Almai A, Rothman DL, Behar KL, Petroff OA et al (2001). Reductions in occipital cortex GABA levels inpanic disorder detected with $1 \mathrm{H}$-magnetic resonance spectroscopy. Arch Gen Psychiatry 58: 556-561.

Goldlust A, Su TZ, Welty DF, Taylor CP, Oxender DL (1995). Effects of anticonvulsant drug gabapentin on the enzymes in metabolic pathways of glutamate and GABA. Epilepsy Res 22: 1-11.

Gomez R, Behar KL, Watzl J, Weinzimer SA, Gulanski B, Sanacora $\mathrm{G}$ et al (2011). Intravenous ethanol infusion decreases human cortical gamma-aminobutyric acid and $\mathrm{N}$-acetylaspartate as measured with proton magnetic resonance spectroscopy at 4 Tesla. Biol Psychiatry 71: 239-246.

Griffith HR, den Hollander JA, Okonkwo OC, O’Brien T, Watts RL, Marson DC (2008). Brain metabolism differs in Alzheimer's disease and Parkinson's disease dementia. Alzheimers Dement 4: 421-427.

Hasler G, van der Veen JW, Tumonis T, Meyers N, Shen J, Drevets WC (2007). Reduced prefrontal glutamate/glutamine and gamma-aminobutyric acid levels in major depression determined using proton magnetic resonance spectroscopy. Arch Gen Psychiatry 64: 193-200.

Hasler G, van der Veen JW, Geraci M, Shen J, Pine D, Drevets WC (2009). Prefrontal cortical gamma-aminobutyric acid levels in panic disorder determined by proton magnetic resonance spectroscopy. Biol Psychiatry 65: 273-275.

Heja L, Barabas P, Nyitrai G, Kekesi KA, Lasztoczi B, Toke O et al (2009). Glutamate uptake triggers transporter-mediated GABA release from astrocytes. PLoS One 4: e7153.

Honarmand A, Safavi M, Zare M (2011). Gabapentin: an update of its pharmacological properties and therapeutic use in epilepsy. J Res Med Sci 16: 1062-1069.

Kammerer M, Rassner MP, Freiman TM, Feuerstein TJ (2011). Effects of antiepileptic drugs on GABA release from rat and human neocortical synaptosomes. Naunyn Schmiedebergs Arch Pharmacol 384: 47-57.

Kassem MN, Bartha R (2003). Quantitative proton short-echo-time LASER spectroscopy of normal human white matter and hippocampus at 4 Tesla incorporating macromolecule subtraction. Magn Reson Med 49: 918-927.

Kegeles LS, Mao X, Stanford AD, Girgis R, Ojeil N, Xu X et al (2012). Elevated prefrontal cortex $\gamma$-aminobutryic acid and glutamate-glutamine levels in schizophrenia measured in vivo with proton magnetic resonance spectroscopy. Arch Gen Psych 69: 449-459.

Kuzniecky R, Ho S, Pan J, Martin R, Gilliam F, Faught E et al (2002). Modulation of cerebral GABA by topiramate, lamotrigine, and gabapentin in healthy adults. Neurology 58: 368-372.

Leach JP, Sills GJ, Butler E, Forrest G, Thompson GG, Brodie MJ (1997). Neurochemical actions of gabapentin in mouse brain. Epilepsy Res 27: 175-180.

Licata SC, Renshaw PF (2010). Neurochemistry of drug action: insights from proton magnetic resonance spectroscopic imaging and their relevance to addiction. Ann NY Acad Sci 1187: $148-171$.

Loscher W, Honack D, Taylor CP (1991). Gabapentin increases aminooxyacetic acid-induced GABA accumulation in several regions of rat brain. Neurosci Lett 128: 150-154.

Mader I, Seeger U, Karitzky J, Erb M, Schick F, Klose U (2002). Proton magnetic resonance spectroscopy with metabolite nulling reveals regional differences of macromolecules in normal human brain. J Magn Reson Imaging 16: 538-546.
Marsman A, van den Heuvel MP, Klomp DW, Kahn RS, Luijten PR, Hulshoff Pol HE (2011). Glutamate in schizophrenia: a focused review and meta-analysis of $1 \mathrm{H}-\mathrm{MRS}$ studies. Schizophr Bull, e-pub ahead of print 11 July 2011.

Mathew NT, Rapoport A, Saper J, Magnus L, Klapper J, Ramadan $\mathrm{N}$ et al (2001). Efficacy of gabapentin in migraine prophylaxis. Headache 41: 119-128.

Mathews GC, Diamond JS (2003). Neuronal glutamate uptake contributes to GABA synthesis and inhibitory synaptic strength. J Neurosci 23: 2040-2048.

Mescher M, Merkle H, Kirsch J, Garwood M, Gruetter R (1998). Simultaneous in vivo spectral editing and water suppression. NMR Biomed 11: 266-272.

O’Gorman RL, Michels L, Edden RA, Murdoch JB, Martin E (2011). In vivo detection of GABA and glutamate with MEGA-PRESS: reproducibility and gender effects. J Magn Reson Imaging 33: 1262-1267.

Petroff OA, Behar KL, Mattson RH, Rothman DL (1996a). Human brain gamma-aminobutyric acid levels and seizure control following initiation of vigabatrin therapy. J Neurochem 67: 2399-2404.

Petroff OA, Hyder F, Mattson RH, Rothman DL (1999). Topiramate increases brain GABA, homocarnosine, and pyrrolidinone in patients with epilepsy. Neurology 52: 473-478.

Petroff OA, Hyder F, Rothman DL, Mattson RH (2000). Effects of gabapentin on brain GABA, homocarnosine, and pyrrolidinone in epilepsy patients. Epilepsia 41: 675-680.

Petroff OA, Hyder F, Rothman DL, Mattson RH (2006). Brain homocarnosine and seizure control of patients taking gabapentin or topiramate. Epilepsia 47: 495-498.

Petroff OA, Rothman DL, Behar KL, Lamoureux D, Mattson RH (1996b). The effect of gabapentin on brain gamma-aminobutyric acid in patients with epilepsy. Ann Neurol 39: 95-99.

Petroff OA, Rothman DL, Behar KL, Mattson RH (1995). Initial observations on effect of vigabatrin on in vivo $1 \mathrm{H}$ spectroscopic measurements of gamma-aminobutyric acid, glutamate, and glutamine in human brain. Epilepsia 36: 457-464.

Petroff OA, Rothman DL, Behar KL, Mattson RH (1996c). Human brain GABA levels rise after initiation of vigabatrin therapy but fail to rise further with increasing dose. Neurology 46: $1459-1463$.

Price RB, Shungu DC, Mao X, Nestadt P, Kelly C, Collins KA et al (2009). Amino acid neurotransmitters assessed by proton magnetic resonance spectroscopy: relationship to treatment resistance in major depressive disorder. Biol Psychiatry 65: 792-800.

Provencher SW (1993). Estimation of metabolite concentrations from localized in vivo proton NMR spectra. Magn Reson Med 30: 672-679.

Rapchuk IL, O'Connell L, Liessmann CD, Cornelissen HR, Fraser JF (2010). Effect of gabapentin on pain after cardiac surgery: a randomised, double-blind, placebo-controlled trial. Anaesth Intensive Care 38: 445-451.

Rothman DL, Behar KL, Hetherington HP, Shulman RG. (1984). Homonuclear $1 \mathrm{H}$ double-resonance difference spectroscopy of the rat brain in vivo. Proc Natl Acad Sci USA 81: 6330-6334.

Rothman DL, Petroff OA, Behar KL, Mattson RH. (1993). Localized $1 \mathrm{H}$ NMR measurements of gamma-aminobutyric acid in human brain in vivo. Proc Natl Acad Sci USA 90: 5662-5666.

Sanacora G, Gueorguieva R, Epperson CN, Wu YT, Appel M, Rothman DL et al (2004). Subtype-specific alterations of gammaaminobutyric acid and glutamate in patients with major depression. Arch Gen Psychiatry 61: 705-713.

Sanacora G, Mason GF, Rothman DL, Behar KL, Hyder F, Petroff OA et al (1999). Reduced cortical gamma-aminobutyric acid levels in depressed patients determined by proton magnetic resonance spectroscopy. Arch Gen Psychiatry 56: 1043-1047.

Sanacora G, Mason GF, Rothman DL, Krystal JH (2002). Increased occipital cortex GABA concentrations in depressed patients after 
therapy with selective serotonin reuptake inhibitors. $A m \mathrm{~J}$ Psychiatry 159: 663-665.

Sanacora G, Zarate CA, Krystal JH, Manji HK (2008). Targeting the glutamatergic system to develop novel, improved therapeutics for mood disorders. Nat Rev Drug Discov 7: 426-437.

Silverman RB, Adruszkiewicz R, Nanavati SM, Taylor CP, Vartanian MG (1991). 3-Alkyl-4 aminobutyric acids: the first class of anticonvulsant agent that activates L-glutamic acid decarboxylase. J Med Chem 34: 2295-2298.

Solinas M, Ferre S, You ZB, Karcz-Kubicha M, Popoli P, Goldberg SR (2002). Caffeine induces dopamine and glutamate release in the shell of the nucleus accumbens. J Neurosci 22: 6321-6324.
Sutoo D, Akiyama K, Yabe K (2000). Quantitative maps of GAbAergic and glutamatergic neuronal systems in the human brain. Hum Brain Mapp 11: 93-103.

Taylor CP, Vartanian MG, Andruszkiewicz R, Silverman RB (1992). 3-Alkyl GABA and 3-alkylglutamic acid analogues: two new classes of anticonvulsant agents. Epilepsy Res 11: 103-110.

Terpstra M, Ugurbil K, Gruetter $\mathrm{R}$ (2002). Direct in vivo measurement of human cerebral GABA concentration using MEGA-editing at 7 Tesla. Magn Reson Med 47: 1009-1012.

Tkac I, Starcuk Z, Choi IY, Gruetter R (1999). In vivo 1H NMR spectroscopy of rat brain at $1 \mathrm{~ms}$ echo time. Magn Reson Med 41: 649-656. 